\title{
Correction to: Synthesis and electrochemical properties of calcium cobaltate as novel anode material
}

\author{
Xiao Chen ${ }^{1}$ (D), Shiyun Zhou ${ }^{2, *}$, and Chuanqi Feng ${ }^{1, *}$ \\ ${ }^{1}$ Hubei Collaborative Innovation Center for Advanced Organic Chemical Materials \& Ministry-of-Education Key Laboratory for \\ Synthesis and Applications of Organic Functional Molecules, Hubei University, Wuhan, People's Republic of China \\ ${ }^{2}$ School of Electronic and Information Engineering, Anshun University, Anshun, People's Republic of China
}

Published online:

6 January 2022

(C) Springer Science+Business

Media, LLC, part of Springer

Nature 2022

Correction to: Journal of Materials Science: Materials in Electronics (2020) $31: 16,020-16,026$ https://doi.org/10.1007/s108 54-020-04164-2

After the publication of our article [1], it was brought to our attention that the legend of Fig. 3 should have included "Figs. $3 a$ and $3 b$ are adapted with permission from J. Phys. Soc. Jpn. 71, 491 (2002). Copyrighted by the Physical Society of Japan". We apologize for this omission.

\section{Reference}

1. X. Chen, S. Zhou, C. Feng, Synthesis and electrochemical properties of calcium cobaltate as novel anode material. J. Mater. Sci. 31, 16020-16026 (2020). https://doi.org/10.100 7/s10854-020-04164-2

Publisher's Note Springer Nature remains neutral with regard to jurisdictional claims in published maps and institutional affiliations.

The original article can be found online at https:/ / doi.org/10.1007/s10854-020-04164-2.

Address correspondence to E-mail: asxyzsy@163.com; cfeng@hubu.edu.cn 